\title{
Caracterización de las condiciones clínicas y parámetros ecocardiográficos de la presión pulmonar en caninos con enfermedad valvular degenerativa
}

\author{
Characterization of the clinical conditions and echocardiographic parameters of \\ pulmonary pressure in dogs with degenerative valvular disease
}

\author{
Paula A. Muñoz-Giraldo1, Leonardo Gómez-Duarte ${ }^{2,3}$
}

\section{Resumen}

La hipertensión pulmonar (HP) es común en perros con enfermedad valvular degenerativa (EVD) y es causada por un aumento de la presión auricular izquierda y presión venosa pulmonar. La cateterización cardíaca es considerada el estándar de oro para el diagnóstico de la HP. La regurgitación tricúspidea (RT), medida por ecocardiografía Doppler, es comúnmente usada en la clínica para determinar el gradiente de presión (GP) de RT reflejando la presión arterial pulmonar (PAP) sistólica. Con el objetivo de caracterizar los parámetros ecocardiográficos de presión pulmonar en caninos con EVD, se revisaron 161 estudios ecocardiográficos y las respectivas historias clínicas de los pacientes que ingresaron a la Unidad de Cardiología Veterinaria en la ciudad de Bogotá en un periodo de 8 meses (noviembre de 2016 y junio de 2017). Se incluyeron todos los pacientes con diagnóstico de EVD, se catalogaron dentro de la clasificación del American College Veterinary Internal Medicine (ACVIM), y se evaluaron algunos criterios indispensables para pertenecer al grupo de estudio. Ninguno de los pacientes presentaba enfermedad primaria o secundaria de origen cardiopulmonar, diferente a la ya mencionada. Se tomaron parámetros ecocardiográficos asociados a la presentación de HP y se realizaron análisis estadísticos para determinar la frecuencia de presentación, relevancia e importancia de cada uno de estos parámetros con el desarrollo de la enfermedad. En conclusión, el mayor porcentaje de pacientes diagnosticados con HP se encontraban clasificados como B2 dentro del ACVIM, lo que sugiere que este grupo de pacientes tiene una mayor posibilidad de presentar HP y, por lo tanto, una mayor posibilidad de congestión. No se encontraron datos relevantes con respecto a la altura de la ciudad (>2600 msnm).

Palabras clave: caninos; ecocardiografía; enfermedad valvular degenerativa; hipertensión pulmonar

\footnotetext{
${ }^{1}$ Facultad de Ciencias Agrarias, Universidad de Antioquia, Medellín, Colombia

${ }^{2}$ Unidad de Cardiología Veterinaria, Bogotá, Colombia

E-mail: olgomezd@gmail.com
}

Recibido: 11 de mayo de 2018

Aceptado para publicación: 22 de noviembre de 2018 
Pulmonary hypertension (PH) is common in dogs with degenerative valvular disease (DVD) and is caused by an increase in left atrial pressure and pulmonary venous pressure. Cardiac catheterization is considered the gold standard for the diagnosis of PH. Tricuspid regurgitation (TR), measured by Doppler echocardiography, is commonly used in the clinic to determine the pressure gradient (PG) of TR reflecting pulmonary arterial systolic pressure (PAP). For the characterization of the echocardiographic parameters of pulmonary pressure in canines with DVD, 161 echocardiographic cases and the respective clinical histories of the patients admitted to the Veterinary Cardiology Unit of the city of Bogotá in a period of 8 months (November 2016 - June 2017) were evaluated. All patients diagnosed with DVD were grouped within the classification of the American College Veterinary Internal Medicine (ACVIM), and some essential criteria were evaluated to assign patients to the study group. None of the patients had a primary or secondary disease of cardiopulmonary origin, different from the one already mentioned. Echocardiographic parameters associated with the presentation of $\mathrm{PH}$ were taken and statistical analyses were carried out to determine the frequency of presentation, relevance and importance of each of these parameters with the development of the disease. In conclusion, the highest percentage of patients diagnosed with $\mathrm{PH}$ were classified as B2 within the ACVIM, which suggests that this group of patients has a greater possibility of presenting $\mathrm{PH}$ and, therefore, a greater possibility of congestion. No relevant data were found regarding the altitude of the city ( $>2600 \mathrm{~m}$ above the sea level).

Key words: canine; echocardiography; pulmonary hypertension; valvular degenerative disease

\section{INTRODUCCIÓN}

La hipertensión pulmonar (HP) es común en los seres humanos con insuficiencia cardíaca, fisiopatológicamente relevante y altamente pronosticable (Guazzi y Naeije, 2017). Se define por un aumento en la presión arterial pulmonar (PAP) sistólica (PAPs) y/o diastólica (PAPd), que puede conducir al desarrollo de insuficiencia cardíaca congestiva del lado derecho (Chetboul, 2016). Se caracteriza por un aumento en la poscarga del ventrículo derecho (VD) (Lankhaar et al., 2008) y un aumento en la resistencia vascular pulmonar (RVP) (Fisher et al., 2009). El VD puede inicialmente adaptarse a esta situación, pero la mayoría de los pacientes mueren a causa de la insuficiencia cardíaca derecha (Lankhaar et al., 2008).
Los valores normales de PAP en el perro despierto varían de 15 a $25 \mathrm{mmHg}$ en la sístole, de 5 a $10 \mathrm{mmHg}$ en la diástole y de 15 mmHg en la PAP media, los cuales pueden aumentar moderadamente durante el ejercicio físico o a gran altura (Chetboul, 2016). La HP sistólica generalmente se considera leve, moderada o severa cuando las PAP son $\leq 50,51-75$, y $>75 \mathrm{mmHg}$, respectivamente (Mikawa et al., 2015; Chetboul, 2016).

Aunque la verdadera incidencia de HP en los perros con insuficiencia cardíaca izquierda es desconocida (Stepien, 2009), un estudio sobre Enfermedad Valvular Degenerativa (EVD) en perros adultos encontró una prevalencia de HP de $14 \%$, y que aumenta con el tamaño de la regurgitación de la válvula mitral (VM) (Serres et al., 2006). Así mismo, Borgarelli et al. (2004) reporta- 
ron que $31 \%$ de perros adultos con EVD presentaban HP. Una disfunción cardíaca del lado izquierdo parece ser la causa más común de HP diagnosticada clínicamente en perros (Ray et al., 2007; Stepien, 2009; Guglin y Khan, 2010).

La EVD es causada por la degeneración mixomatosa progresiva de la válvula mitral, ya sea en solitario o junto con la válvula tricúspide (Borgarelli et al., 2008). Se caracteriza por los cambios en los componentes celulares, así como la matriz intercelular (Atkins et al., 2009; Bonagura y Schober, 2009) del aparato valvular (incluyendo las valvas y cuerdas tendinosas). El prolapso de la válvula mitral es una complicación común de la EVD y representa una característica destacada en algunas razas (Atkins et al., 2009). La EVD afecta con mayor frecuencia la válvula atrioventricular izquierda, seguida de la válvula atrioventricular derecha (Häggström et al., 2005; Atkins et al., 2009), aunque pueden estar afectadas tanto la válvula mitral como la tricúspide a la vez (Häggström et al., 2005; Borgarelli et al., 2008). La causa de EVD es hereditaria (Olsen et al., 2003; Atkins et al., 2009).

Se estima que el $10 \%$ de los perros presentados por primera vez a la consulta veterinaria tiene una enfermedad cardíaca, siendo la EVD la enfermedad cardíaca más común (Borgarelli et al., 2008; Atkins et al., 2009). Pedersen et al. (1999) y Olsen et al. (2003) señalan como factores de riesgo para la progresión de la EVD a la edad, el sexo, la intesidad del soplo en el corazón, el grado de prolapso en la válvula, la gravedad de las lesiones en la válvula y el grado de regurgitación en la válvula mitral.

La enfermedad es 1.5 veces más común en machos que en hembras, y es mayor en razas pequeñas $(<20 \mathrm{~kg})$ (Atkins et al., 2009). En perros de talla pequeña ocurre una progresión lenta durante años (Atkins et al., 2009; Bonagura y Schober, 2009), pero la progresión es imprevisible en la mayoría de los perros que experimentan la aparición de un soplo reconocible de regurgitación de la válvula mitral años antes de la aparición clínica de la insuficiencia cardíaca (Atkins et al., 2009). La progresión de la EVD en perros de razas grandes parece ser más rápida que la observada en perros de razas pequeñas (Atkins et al., 2009). El Cavalier King Charles Spaniel (CKCS) está predispuesto a desarrollar EVD a una edad relativamente joven, pero la evolución de la enfermedad es similar al de otras razas pequeñas (Atkins et al., 2009; Häggström et al., 2009).

Los signos clínicos de la HP pueden ser sutiles y similares a los signos de la insuficiencia cardíaca congestiva (ICC) izquierda (Stepien, 2009). La ascitis puede ser la anomalía más evidente, pero los signos clínicos más frecuentes incluyen anomalías respiratorias inespecíficas, como tos, taquipnea, dificultad respiratoria y síncope (MacDonald y Johnson, 2005).

La ecocardiografía Doppler es un método no invasivo y que permite diagnosticar la HP (Kellum y Stepien, 2007; Chiavegato et al., 2009) en animales conscientes. La presión pulmonar sistólica puede estimarse mediante la medición de la velocidad de regurgitación tricúspidea (RT), la presión diastólica evaluando el gradiente de presión pulmonar (Kellum y Stepien, 2007; Chiavegato et al., 2009) y el gradiente de presión (GP) transvalvular tricúspide utilizando el pico de velocidad de RT en la ecuación modificada de Bernoulli (Chiavegato et al., 2009; Brown et al., 2010).

En el presente estudio se caracterizaron algunas variables ecocardiográficas de los pacientes con HP secundaria a EVD de la ciudad de Bogotá, ya que la HP puede ser una consecuencia frecuente de la EVD.

\section{Materiales y Métodos}

Se realizó un estudio retrospectivo donde se revisaron 161 historias clínicas de estudios ecocardiográficos de pacientes que in- 
gresaron a la Unidad de Cardiología Veterinaria (UCV) de la ciudad de Bogotá entre el 1 de noviembre de 2016 y el 30 de junio de 2017. La ciudad está ubicada entre 2600 y $3000 \mathrm{msnm}$. El estudio tuvo el aval del Comité de Ética para Experimentación con Animales de la Universidad de Antioquia.

\section{Pacientes}

Se incluyeron a todos los caninos pacientes con EVD ( $n=89)$. Todos fueron incluidos dentro de la clasificación del American College of Veterinary Internal Medicine (ACVIM) (Atkins et al., 2009; Gómez, 2011). Se excluyeron los perros que presentaban enfermedades cardíacas congénitas o desórdenes cardiovasculares adquiridos que directa o indirectamente afectan las válvulas cardíacas o su función, como la endocarditis bacteriana o cardiomiopatía dilatada idiopática (CDI), pacientes con presencia de alguna enfermedad pulmonar primaria, así como los que presentaban dirofilariasis. Se excluyeron específicamente los perros de West Highland White Terrier, dado que pueden presentar una fibrosis pulmonar idiopática crónica (Webb y Armstrong, 2002).

\section{Evaluación Ecocardiográfica}

Se realizaron exámenes ecocardiográficos con un equipo de ultrasonido Mindray DCN3-VET dotado con transductores phased-array de 3.0-7.0 MHz. Los pacientes se sometieron a un examen ecocardiográfico completo según protocolo previamente establecido (Serres et al., 2006), que incluyó ecocardiografía transtorácica bidimensional (2D), modo M, modo espectral pulsado y continuo, y Doppler color. Se realizaron evaluaciones en pacientes no sedados, en decúbito lateral izquierda y derecha y vistas ecocardiográficas eje corto, eje largo y apical.

Los estudios ecocardiográficos fueron realizados por un experto en el área de cardiología veterinaria. Todos los datos fueron capturados digitalmente para su análisis posterior. Se tuvo cuidado de alinear el jet de RT lo más paralelo posible al plano del cursor de ultrasonido (Visser et al., 2016). Las variables evaluadas fueron aquellas que la literatura científica (Borgarelli et al., 2008; Stepien, 2009; Chetboul, 2016) y la experiencia clínica y ecocardiográfica de los investigadores sugieren que existe grados variables de HP.

Las mediciones del ventrículo izquierdo (VI) se obtuvieron en una vista paraesternal de eje largo (Lang et al., 2015; Tidholm et $a l ., 2015)$. La relación de diámetro del atrio izquierdo (AI) a la aorta (Ao) $(\mathrm{AI} / \mathrm{Ao})$ se midió según Hansson et al. (2002). La medición se realizó en modo $2 \mathrm{D}$ en una vista en eje corto. Una relación $\mathrm{AI} / \mathrm{Ao}<1.6$ se consideró normal (Baron-Toaldo et al., 2016).

Desde una vista paraesternal derecha, mediante el método 2D utilizando la visión de eje corto transaórtico, se obtuvieron mediciones diastólicas de las dimensiones internas de la arteria pulmonar principal (MPA, por sus siglas en inglés) y Ao. El diámetro de MPA se midió justo debajo de la válvula pulmonar cerrada y el diámetro aórtico se midió en la misma vista y se calculó la relación MPA/Ao (Serres et al., 2007). El flujo de la arteria pulmonar se midió y registró con Doppler de onda pulsada desde la vista del eje corto paraesternal derecho, usando Imagen Doppler color para guiar la colocación del volumen de muestra $(1-3 \mathrm{~mm})$ centralmente entre las valvas de la válvula pulmonar abierta (Visser et al., 2016). La relación MPA/Ao anómala se considera un signo temprano e indirecto de HP y su rango es: 0.8-1.15 (Chetboul, 2016).

La presencia de regurgitación de la válvula mitral se evaluó mediante Doppler color en el eje largo paraesternal derecho y vistas apicales izquierdas. La vista apical izquierda de cuatro cámaras es la mejor vista para registrar los flujos atrioventriculares. El flujo transmitral se caracteriza por tener dos picos de velocidad: un gran pico diastólico tempra- 
no (onda E), que corresponde al llenado ventricular rápido, $\mathrm{y}$ un pico pequeño (onda A) que acompaña a la contracción auricular. El valor máximo de la onda $\mathrm{E}$ es de $87 \pm 13$ $\mathrm{cm} / \mathrm{s}$ (Chetboul, 2016). La variable hemodinámica EMax para este estudio fue la velocidad máxima de la onda $\mathrm{E}$ del flujo transmitral.

El flujo pulmonar tiene una forma simétrica y generalmente se registra desde una ventana paraesternal derecha en una vista transaórtica en eje corto. Para el flujo pulmonar, las mediciones incluyen, entre otras, integral de velocidad-tiempo (IVT) que se obtiene midiendo el área bajo la curva del espectro del flujo pulmonar, y los Tiempos de Eyección (TE) y Aceleración (TA), a partir de los cuales se obtiene la relación TA:TE (Chetboul, 2016). El TA se midió desde el comienzo del flujo Doppler de onda pulsada hasta la velocidad máxima de flujo. El TE se midió desde el inicio hasta el final de la señal de flujo Doppler de onda pulsada de la arteria pulmonar, y se calculó una relación TA:TE (Schober et al., 2006). Se considera un valor normal de TA: $>58 \mathrm{~ms}$ y la relación TA:TE $>0.31$ (Stepien, 2009).

La velocidad máxima del jet de RT se obtuvo mediante ecografía 2D/Doppler de onda continua guiada por Doppler color y se midió desde la vista que permitía el perfil del jet de RT más limpio y con la mejor alineación respecto a la dirección de la regurgitación. La velocidad del jet de RT solo se midió cuando estaba presente un espectro de flujo completo del jet y la velocidad máxima se visualizaba claramente (Visser et al., 2016).

El gradiente de presión transvalvular tricúspideo se puede estimar utilizando la velocidad pico de RT en la ecuación de Bernoulli modificada: $\mathrm{GP}=4 \mathrm{x}$ [velocidad máxima $\mathrm{RT}]^{2}$ (Fisher et al., 2009), por lo tanto el RT-PAPs por encima de $30 \mathrm{mmHg}$ se considera sugerente de HP (Chiavegato et al., 2009; Stepien, 2009).

\section{Diseño Estadístico}

Se realizó estadística descriptiva de los datos demográficos (variables categóricas) agrupándolos como frecuencias, proporciones y variables continuas resumidas como medias o medianas con sus medidas de dispersión. Se realizó un análisis bivariado para la asociación entre la edad y la hipertensión pulmonar usando el Fishers exact test. Se realizó el análisis de normalidad de los datos usando las pruebas de D'Agostino's Ksquared y Shapiro-Wilk Test.

Se realizaron pruebas paramétricas $(\mathrm{t}$ Student) o no paramétricas (U-Mann Whitney) según las diferencias en variables continuas para encontrar diferencias entre los pacientes con y sin HP. Los valores de $p<0.05$ se consideraron estadísticamente significativos. Los análisis se presentaron en diagramas de cajas y bigotes. Los análisis se realizaron usando el paquete estadístico Prism ${ }^{\circledR} 7.01$ para $\mathrm{Mac}^{\mathrm{TM}}$.

\section{Resultados y Discusión}

La población de estudio $(\mathrm{n}=89)$ estuvo conformada por $61.8 \%$ de machos, lo cual concuerda con otros reportes que demuestran que la EVD es 1.5 veces más común en

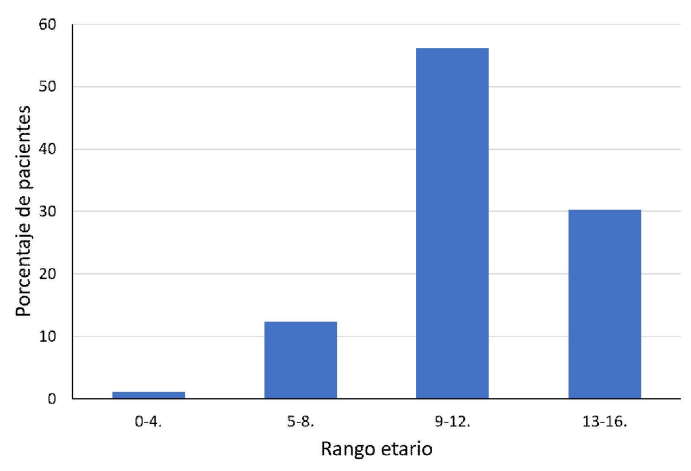

Figura 1. Rango de edad de los pacientes con Enfermedad Valvular Degenerativa (EVD) 


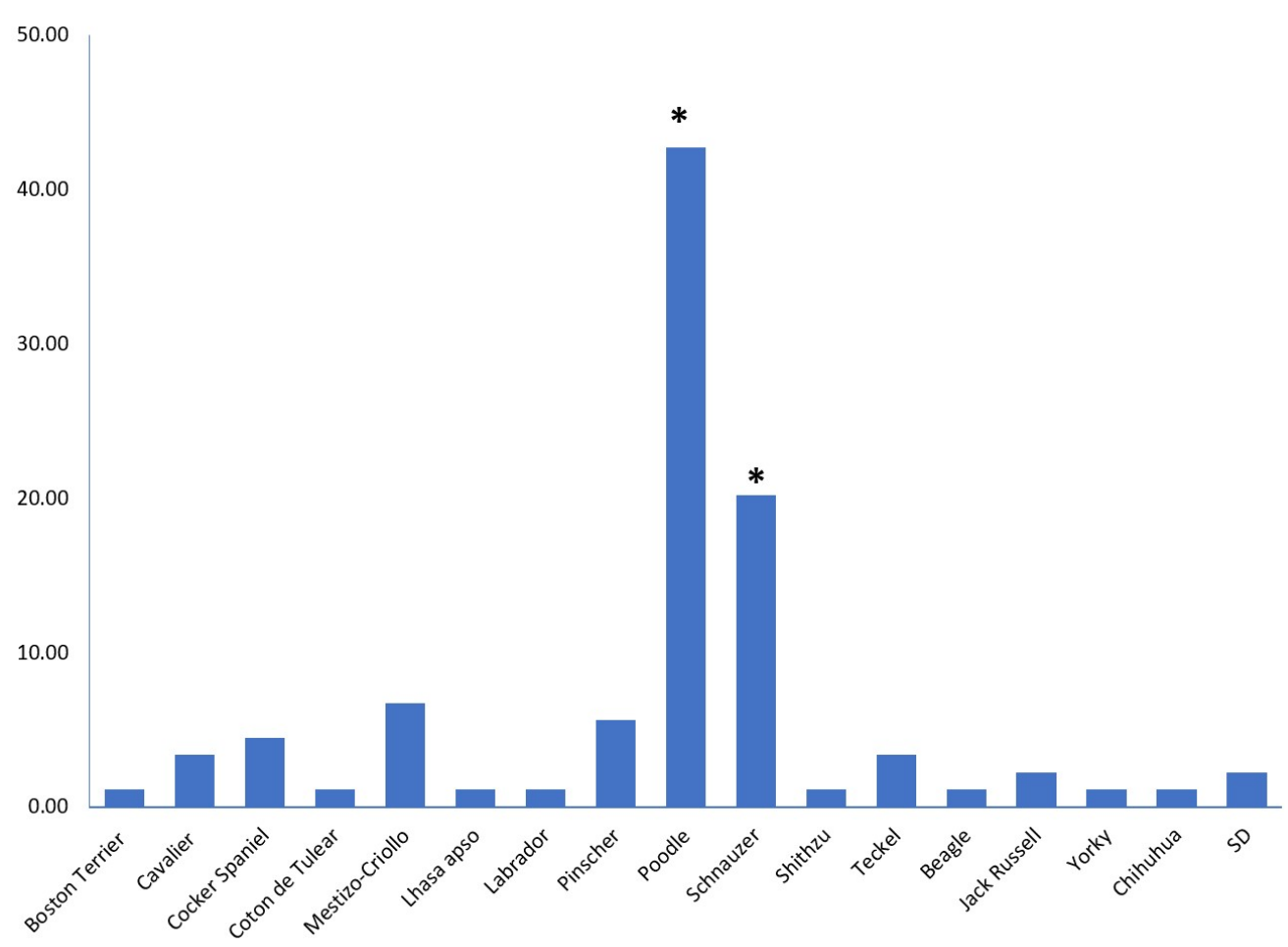

Figura 2. Distribución porcentual por raza de los pacientes con Enfermedad Valvular Degenerativa (EVD)

machos que en hembras (Atkins et al., 2009; Garncarz et al., 2013). Así mismo, el 56.2\% de los pacientes estaban dentro del rango de 9 a 12 años, seguidos por el grupo de 13-16 años y en menor proporción los pacientes menores de 8 años (Figura 1). En el estudio de Borgarelli et al. (2008) con 722 historias clínicas de EVD, la edad media de los perros fue de $10.6 \pm 2.6$, mientras que en este estudio fue de $11.3 \pm 2.4$ años (4-16 años). Otros autores indican que la prevalencia de EVD aumenta marcadamente con la edad, con alguna evidencia de lesiones en la necropsia, hasta un 85\%, a los 13 años (Atkins et al., 2009).

Se ha descrito que la presentación clínica de la EVD ocurre con mayor frecuencia en animales de razas pequeñas como Poodle estándar y miniatura, Schnauzer, Pinscher, y mestizos menores de $15 \mathrm{~kg}$. En algunos casos se puede encontrar en razas como La- brador o en mestizos de talla grande (Ware, 2007). En el presente estudio se encontró mayormente en los pacientes de razas Poodle (42.7\%) y Schnauzer (20.2\%) (Figura 2), lo cual coincide con Atkins et al. (2009).

\section{Clasificación ACVIM}

El Colegio Americano de Medicina Interna Veterinaria publicó en 2009 un documento de consenso en el cual se proponen los lineamientos para el diagnóstico y tratamiento de la EVD en perros, el cual incluye cuatro etapas de clasificación (Atkins et al., 2009; Gómez, 2011). Los pacientes del presente estudio se ubicaron en las tres primeras categorías, siendo la clase B la de mayor presentación (Figura 3). No obstante, Vezzosi et al. (2017) reportaron que la mayoría de los pacientes estuvieron clasificados en los estadios B1, B2 y C, mientras que Borgarelli et al. (2015) encontraron que la HP fue más 


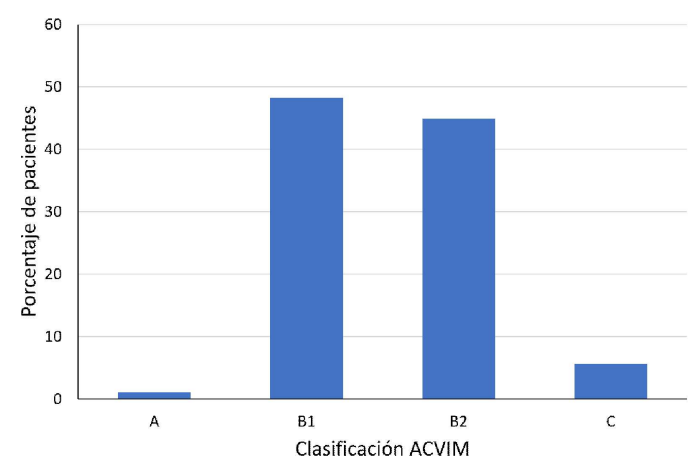

Figura 3. Clasificación porcentual de 89 pacientes caninos con Enfermedad Valvular Degenerativa (EVD), según el Colegio Americano de Medicina Interna Veterinaria (ACVIM)

frecuente en pacientes en etapa C. Es importante resaltar que aunque el porcentaje de pacientes en clase $\mathrm{C}$ fue bajo en el presente estudio, son pacientes con mayor riesgo de presentar signos clínicos de congestión y posible falla cardíaca (Atkins et al., 2009).

En el presente estudio, $13.4 \%$ de pacientes presentaron HP y $86.6 \%$ no lo presentaron, lo cual concuerda con los reportes de Borgarelli et al. (2015) y Mikawa et al. (2015) donde se indican que el 39 y $21 \%$ de pacientes fueron diagnosticados con HP.

\section{Medidas Ecocardiográficas}

\section{Medidas anatómicas}

Similar a lo reportado en la literatura (Cornell et al., 2004; Borgarelli et al., 2008; Chetboul, 2016), el Cuadro 1 muestra que los pacientes con EVD que no tenían HP mantuvieron los valores promedio dentro de los rangos que se reportan como normales para DIVId (dimensión del ventrículo izquierdo en diástole), AI/Ao y MPA/Ao.

Reportes en la literatura para pacientes con EVD indican un valor promedio de 27 mm del DIVId (Cornell et al., 2004), el cual es menor que el valor de $30.8 \mathrm{~mm}$ del presente estudio, para el rango promedio de referencia para el peso, considerando que los pacientes en este estudio estaban por debajo de los $15 \mathrm{~kg}$, a excepción de un Labrador (32 $\mathrm{kg}$ ). Esto sugiere un agrandamiento del ventrículo e indica cambios hemodinámicos significativos con remodelación cardíaca (Gómez, 2011). Las medidas anatómicas en pacientes con EVD y con HP se muestran, asimismo, en el Cuadro 1.

La relación MPA/Ao es un signo temprano e indirecto de HP (Chetboul, 2016; Visser et al., 2016). En el Cuadro 1 se observa que el valor promedio de los perros con HP se mantuvo dentro del rango normal (0.81.15), lo cual coincide con lo reportado en la literatura que indica que la relación MPA/Ao tiene una sensibilidad del $45 \%$ para la detección de HP (Chetboul, 2016). En el presente estudio, solo el $42.8 \%$ de los pacientes con HP superaba el rango máximo de la realción MPA/Ao.

En el estudio de Borgarelli et al. (2008) se encontró que la relación $\mathrm{AI} / \mathrm{Ao}$ tuvo una significación estadística cuando solo se incluyeron las muertes relacionadas con causas cardíacas; es decir, que servía como predictor de la supervivencia en pacientes con EVD. En el presente estudio no se valoró la supervivencia de los pacientes; sin embargo, el valor promedio de la relación $\mathrm{AI} / \mathrm{Ao}$ de los pacientes con HP (Cuadro 1) sobrepasó el valor reportado como normal $(>1.7)$, el cual está estadísticamente asociado como causa de muerte (Borgarelli et al., 2008). Esto significa que puede ser una medida útil para determinar el pronóstico de los pacientes.

No se encontró diferencia estadística entre los valores de DIVId de pacientes con y $\sin$ HP (Figura 4A). Esto es importante porque el aumento del valor de DIVId sugiere el agrandamiento del ventrículo e indica cambios hemodinámicos significativos con la remodelación cardíaca (Gómez, 2011). Por otro lado, el valor de la relación MPA/Ao y la relación $\mathrm{AI} / \mathrm{Ao}$ en pacientes con HP fue 


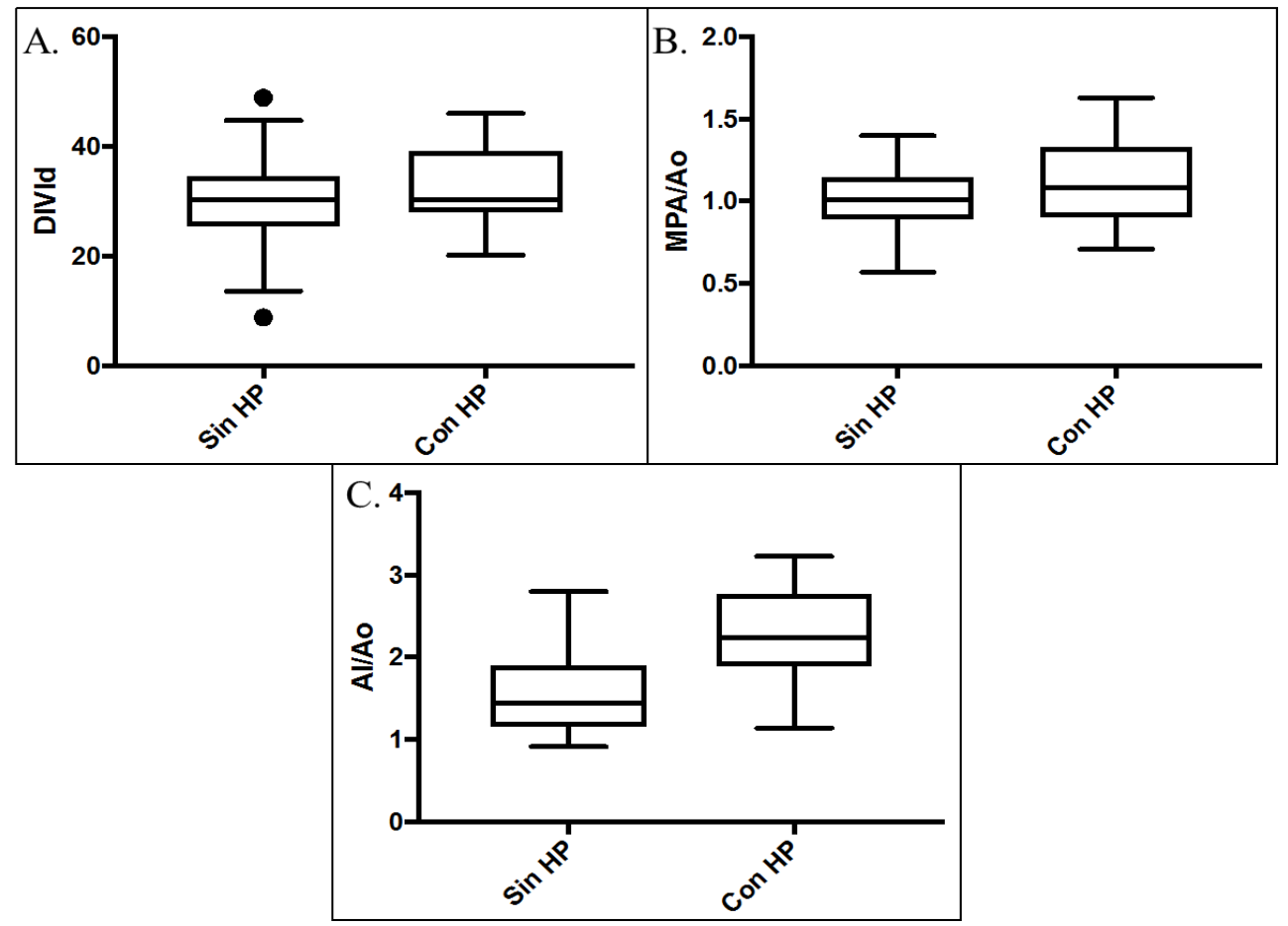

Figura 4. Medidas anatómicas en pacientes con y sin hipertensión pulmonar (HP). A. Valor de $\operatorname{DIVId}(\mathrm{p}=0.3067) ; \mathrm{B}$. Valor del MPA/Ao $(\mathrm{p}=0.0337) ; \mathrm{C}$. Valor de $\mathrm{AI} / \mathrm{Ao}(\mathrm{p}=<0.0001)$



Figura 5. Medidas hemodinámicas en pacientes con y sin hipertensión pulmnar (HP). A. Valor de EMax $(\mathrm{p}=<0.0077)$. B. Valor de TA $(\mathrm{p}=<0.0011)$. C. Valor de la IVT $(\mathrm{p}=<0.0032)$. D. Valor de TA:TE $(\mathrm{p}=<0.8676)$ 
Cuadro 1. Medidas anatómicas en pacientes con enfermedad valvular degenerativa (EVD) con y sin hipertensión pulmonar (HP)

\begin{tabular}{lcccccccc}
\hline & \multicolumn{3}{c}{ Sin hipertensión arterial } & \multicolumn{3}{c}{ Con hipertensión arterial } \\
\cline { 2 - 9 } & Media & DE & Máx & Mín & Media & DE & Máx & Mín \\
\hline DIVId & 30.8 & 7.1 & 48.9 & 8.8 & 32.2 & 7.5 & 46 & 20.2 \\
MPA/Ao & 1 & 0.20 & 1.4 & 0.57 & 1.13 & 0.26 & 1.63 & 0.71 \\
AI/Ao & 1.53 & 0.45 & 2.8 & 0.91 & 2.26 & 0.63 & 3.23 & 1.14 \\
\hline
\end{tabular}

DIVId: Dimensión del ventrículo izquierdo en diástole

Al/Ao: Relación del diámetro del atrio izquierdo (Al) a la aorta (Ao)

MPA/Ao: Relación entre el diámetro de la arteria pulmonar principal (MPA) al diámetro de la aorta (Ao)

Cuadro 2. Medidas hemodinámicas en pacientes con enfermedad valvular degenerativa (EVD) con y sin hipertensión pulmonar (HP)

\begin{tabular}{lllllllll}
\hline & \multicolumn{3}{c}{ Sin hipertensión arterial } & \multicolumn{4}{c}{ Con hipertensión arterial } \\
\cline { 2 - 8 } & \multicolumn{1}{c}{ Media } & \multicolumn{1}{c}{ DE } & \multicolumn{1}{c}{ Máx } & Mín & Media & \multicolumn{1}{c}{ DE } & Máx & Mín \\
\hline EMax & 102 & 32.03 & 174.8 & 53.43 & 144.7 & 74.57 & 363.5 & 47.21 \\
IVT & 9.73 & 16.78 & 40.3 & 3.38 & 8.54 & 9.14 & 39 & 2.64 \\
TA & 70.93 & 21.5 & 116.0 & 32.0 & 54 & 19.45 & 100 & 30 \\
TA:TE & 0.47 & 0.08 & 0.73 & 0.23 & 0.47 & 0.11 & 0.73 & 0.23 \\
RT-PAPs & No & No & No & No & 40.31 & 12.49 & 60.1 & 18.5 \\
& tiene & tiene & tiene & tiene & & & & \\
\hline
\end{tabular}

EMax: Velocidad máxima de la onda E del flujo transmitral; IVT: Integral de Velocidad-Tiempo; TA: Tiempo de Aceleración; TA:TE: Relación entre TA y Tiempo de Eyección (TE); RT-PAPs: Regurgitación tricuspidea-Presión Arterial Pulmonar sistólica

significativamente mayor que en pacientes sin $\mathrm{HP}(\mathrm{p}=0.0337$ y $\mathrm{p}=<0.0001$, respectivamente) (Figura 4B y 4C). La diferencia estadística para $\mathrm{AI} /$ Ao fue igualmente reportada por Borgarelli et al. (2008).

\section{Medidas hemodinámicas}

Los pacientes que no presentaron HP mantuvieron los valores promedio dentro de los rangos que la literatura reporta como normales (Borgarelli et al., 2008; Stepien, 2009; Chetboul, 2016) (Cuadro 2). Se ha reportado que la velocidad máxima del llenado transmitral temprano depende del volumen y la presión auricular; en consecuencia, el aumento de EMax puede considerarse como un indicador del aumento de la presión de la aurícula izquierda (Borgarelli et al., 2008). Por lo tanto, los datos de este estudio son consistentes con los informes de estudios en

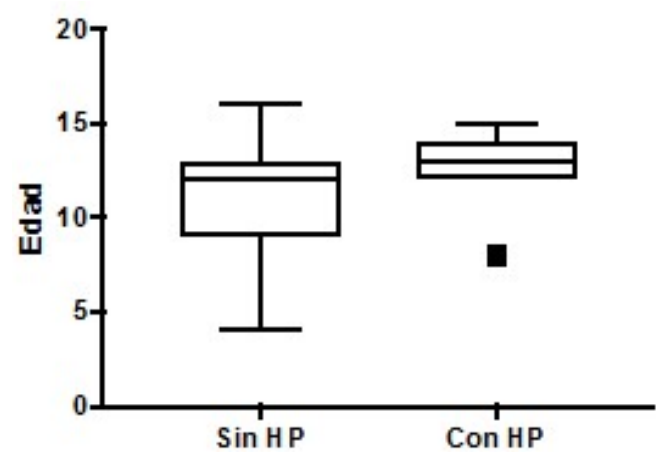

Figura 6. Distribución de la edad en pacientes con y sin hipertensión pulmonar (HP) $(\mathrm{p}=0.0061)$ 


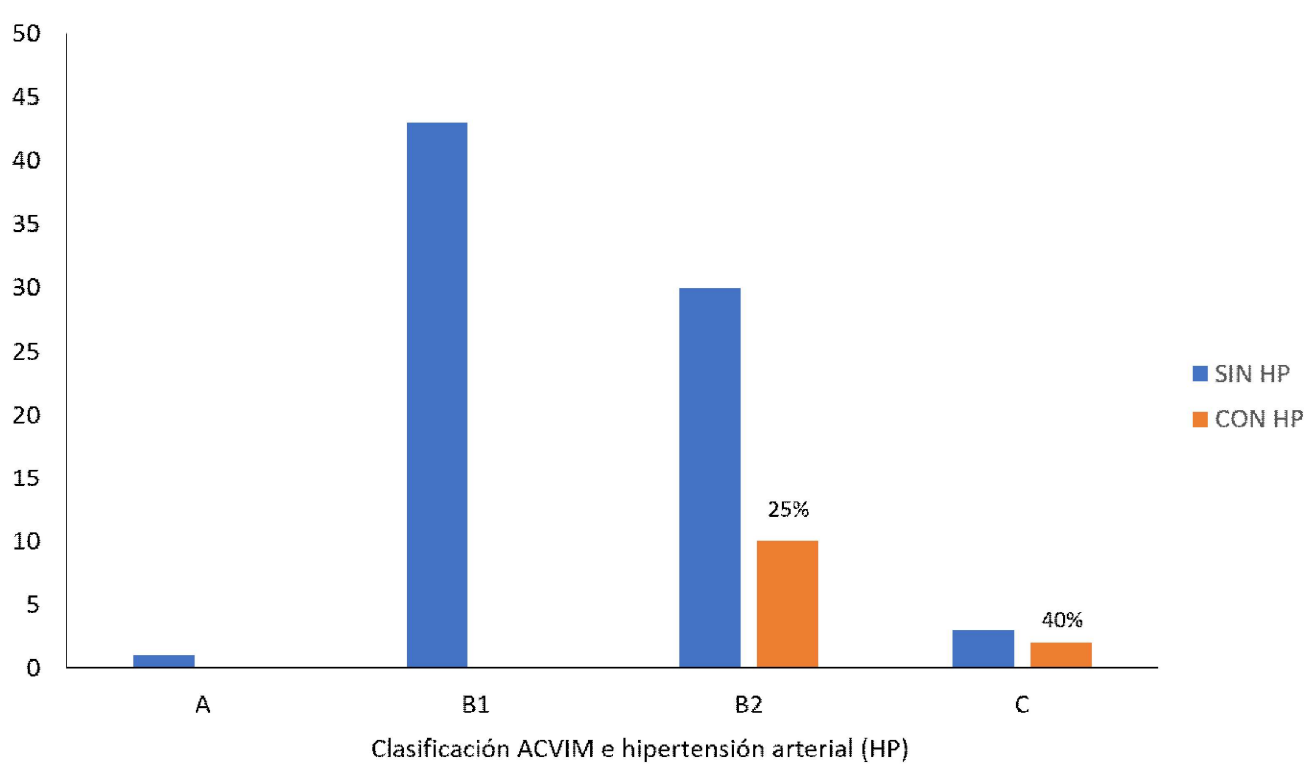

Figura 7. Distribución porcentual de la clasificación del Colegio Americano de Medicina Interna Veterinaria (ACVIM) en pacientes con y sin hipertensión pulmonar (HP)

perros que sugieren que la gravedad de la regurgitación de la válvula mitral es un predictor de mal pronóstico (Borgarelli et al., 2008).

La presencia de HP altera la hemodinámica normal de la válvula pulmonar, por lo que el estudio de los espectros Doppler del flujo pulmonar puede aportar una información adicional diagnóstica y pronóstica en los pacientes con HP, los cuales se clasifican en perfil Tipo I, II y III (Johnson et al., 1999; Talavera y Fernández del Palacio, 2007).

Los intervalos de tiempo sistólicos (ITS), incluido el tiempo de la velocidad de flujo de la arteria pulmonar (AP) hasta el pico (denominado tiempo de aceleración, TA); tiempo de eyección del ventrículo derecho (TE); y el índice de tiempo de aceleración (referido como relación de tiempo de aceleración a tiempo de eyección, TA:TE) son variables ecocardiográficas Doppler que se han utilizado para estimar las presiones de la AP sistólica y diagnosticar la HP en pacientes humanos (Schober y Baade, 2006). En los pacientes del presente estudio con HP se encontraron algunas variables con el valor promedio alterado (Cuadro 2), que coincide con Stepien (2009) quien reporta PAPs $>30$ $\mathrm{mmHg}, \mathrm{TA} \leq 58$ y una relación TA: $\mathrm{TE} \leq 0.31$, que son consistentes con la presencia de HP. Así mismo, Schober et al. (2006) y Tidholm et al. (2015) concluyen que la HP se asocia con un acortamiento de la TA y una disminución de la relación TA:TE.

La presencia de RT permite la estimación exacta de la PAPs (Chiavegato et al., 2009; Fisher, et al., 2009). En el presente estudio, 12 de los 89 pacientes (13.4\%) tenían un reflujo tricuspídeo sugerente de HP. Se debe tener en cuenta que no todos los pacientes humanos con HP desarrollan una regurgitación tricúspide significativa (Mikawa et al., 2015), lo que podría ser significativo en los pacientes de este estudio que tenían algunas variables alteradas y que no presentaban un jet de RT significativa que correspondiera con HP. 
Los resultados muestran significancia estadística para EMax y TA en pacientes con $y \sin \mathrm{HP}(\mathrm{p}=<0.0077$ y $\mathrm{p}=<0.0011$, respectivamente) (Figura 5A y 5B). En la Figura 5C se puede observar datos atípicos para la IVT, que puede ser explicado teniendo en cuenta que los espectros Doppler del flujo pulmonar se pueden clasificar en perfil Tipo I, II y III, ya que la presencia de HP altera la hemodinámica normal de la válvula pulmonar (Talavera y Fernández del Palacio, 2007).

En contraste a lo reportado por Schober et al. (2006), que concluyen que la hipertensión pulmonar se asocia con una disminución de la relación TA:TE, en el presente estudio la relación TA:TE no tuvo una diferencia significativa entre los pacientes con y sin HP (Figura 5D), aunque se puede observar una más amplia distribución de dicha relación en los pacientes con HP.

El factor edad fue significativo entre pacientes con y $\sin$ HP ( $p=0.0061$ ) (Figura 6), donde los pacientes con HP tienen mayor edad que aquellos sin HP; sin embargo, y de acuerdo con los datos que menciona Mikawa et al. (2015), al realizar una prueba de asociación de Fisher entre un paciente adulto ( $>8$ años) y tener HP, el resultado mostró una $\mathrm{p}=0.6895$, lo que indica que no había asociación entre estos dos factores. Esto significa que si bien hay diferencia estadística por edad entre los grupos con y sin HP, tener más de 8 años no podría considerarse un factor de riesgo para el desarrollo de HP en pacientes con EVD.

En la Figura 7 se observa que el mayor porcentaje de pacientes con HP se ubican en Clase B2 y C. Se podría decir que en el presente estudio se tenían 40 pacientes de Clase B2, de los cuales 10 se diagnosticaron con HP para este dato, lo que representa un $25 \%$. Así mismo, en Clase C se tuvo cinco pacientes y dos con HP, lo que representa que $40 \%$ de los pacientes en Clase $\mathrm{C}$ se diagnosticaron con HP. Estos resultados se asemejan a los encontrados por Borgarelli et al. (2015) y Mikawa et al. (2015), donde se evidencia que a mayor es la clasificación ACVIM mayor es el porcentaje de pacientes con HP.
Entre los pacientes diagnosticados con HP, el $64.3 \%$ se clasificaron con HP leve y el $35.7 \%$ con HP moderada, mientras que ningún paciente tuvo un grado de HP severa. Este resultado puede darse debido a una temprana identificación de las alteraciones cardíacas, lo que hace que los propietarios acudan a la consulta cardiológica, permitiendo que con apoyo del cardiólogo veterinario pueda hacerse un adecuado control de la enfermedad y se logre estabilizar el paciente teniendo una mejor calidad de vida.

En la revisión de las historias clínicas se encontró que los propietarios del $50 \%$ de los perros con HP habían reportado episodios de síncope, un $37.5 \%$ tos y otro $37.5 \%$ de intolerancia al ejercicio, los cuales son consistentes con la literatura (Garncarz et al., 2013; Mikawa et al., 2015; Chetboul 2016). Los pacientes de este estudio viven en una zona con altitud mayor a los $2600 \mathrm{msnm}$, lo cual puede inducir hipoxia que contribuye a la vasoconstricción y al aumento de la RVP, que puede contribuir al desarrollo de HP en los perros (Talavera y Fernández del Palacio, 2007; Guazzi y Naeije, 2017). Sin embargo, muchos de los resultados obtenidos son similares a los reportados en pacientes que viven a nivel del mar (Rich, 2001; Talavera y Fernández del Palacio, 2007), los cuales indican que la vascularización pulmonar en la especie canina sufre menos vasoconstricción que otras especies en respuesta a bajas tensiones de oxígeno.

\section{Conclusiones}

- $\quad$ El grupo de pacientes con HP clasificados como ACVIM Clase B2 son los de mayor riesgo de congestión.

- No se encontraron alteraciones relevantes en las medidas ecocardiográficas que sugieran que se encuentran influenciadas por la altura (>2600 msnm), comparados con los valores que se reportan a nivel del mar. 


\section{Literatura Citada}

1. Atkins C, Bonagura J, Ettinger S, Fox $P$, Gordon S, Haggstrom J, Hamlin R, et al. 2009. Guidelines for the diagnosis and treatment of canine chronic valvular heart disease. J Vet Intern Med 23: 1142-1150. doi: 10.1111/j.19391676.2009.0392.x

2. Baron M, Poser H, Menciotti G, Battaia S, Contiero B, Cipone M, Diana $A$, et al. 2016. Utility of tissue doppler imaging in the echocardiographic evaluation of left and right ventricular function in dogs with myxomatous mitral valve disease with or without pulmonary hypertension. J Vet Intern Med 30: 697 705. doi: 10.1111/jvim. 13940

3. Bonagura JD, Schober KE. 2009. Can ventricular function be assessed by echocardiography in chronic canine mitral valve disease? J Small Anim Pract 50: 12-24. doi: 10.1111/j.17485827.2009.00803.x

4. Borgarelli M, Zini E, D'Agnolo G, Tarducci A, Santilli RA, Chiavegato $D$, Tursi M, et al. 2004. Comparison of primary mitral valve disease in German shepherd dogs and in small breeds. J Vet Cardiol 6: 27-34. doi: 10.1016/S17602734(06)70055-8

5. Borgarelli M, Savarino P, Crosara $S$, Santilli RA, Chiavegato D, Poggi M, Bellino $C$, et al. 2008. Survival characteristics and prognostic variables of dogs with mitral regurgitation attributable to myxomatous valve disease. J Vet Intern Med 22: 120-128. doi: 10.1111/j.1939-1676.2007.0008.x

6. Borgarelli M, Abbott J, Braz-Ruivo L, Chiavegato D, Crosara S, Lamb K, Ljungvall I, et al. 2015. Prevalence and prognostic importance of pulmonary hypertension in dogs with myxomatous mitral valve disease. J Vet Intern Med 29: 569-574. doi: 10.1111/jvim. 12564

7. Brown AJ, Davison E, Sleeper MM. 2010. Clinical efficacy of sildenafil in treatment of pulmonary arterial hyper- tension in dogs. J Vet Intern Med 24: 850854. doi: 10.1111/j.1939-1676.2010.0517.x

8. Chetboul V. 2016. Pulmonary arterial hypertension. In: Chetboul V, Bussadori $\mathrm{C}$ (eds). Clinical echocardiography of the dog and cat. USA: Elsevier Masson. $p$ 229-240.

9. Chiavegato D, Borgarelli $M$, D'Agnolo G, Santilli RA. 2009. Pulmonary hypertension in dogs with mitral regurgitation attributable to myxomatous valve disease. Vet Radiol Ultrasoun 50: 253-258. doi: 10.1111/ j.1740-8261.2009.01529.x

10. Cornell CC, Kittleson MD, Della P, Häggström J, Lombard CW, Pedersen HD, Vollmar A, et al. 2004. Allometric scaling of M-mode cardiac measurements in normal adult dogs. J Vet Intern Med 18: 311-321. doi: 10.1111/j.19391676.2004.tb02551.x

11. Fisher MR, Forfia PR, Chamera E, Housten-Harris T, Champion HC, Girgis RE, Corretti MC, et al. 2009. Accuracy of doppler echocardiography in the hemodynamic assessment of pulmonary hypertension. Am J Resp Crit Care 179: 615-621. doi: 10.1164/ rccm.200811-16910C

12. Garncarz M, Parzeniecka-Jaworska M, Jank M, Lój M. 2013. A retrospective study of clinical signs and epidemiology of chronic valve disease in a group of 207 Dachshunds in Poland. Acta Vet Scand 55: 52. doi: 10.1186/ 1751-0147-55-52

13. Gómez L. 2011. Enfermedad valvular degenerativa en perros: actualización en su diagnóstico, tratamiento y pronóstico. Rev Colomb Cienc Pec 24: 201-208.

14. Guazzi M, Naeije R. 2017. Pulmonary hypertension in heart failure: pathophysiology, pathobiology, and emerging clinical perspectives. J Am Coll Cardiol 69: 1718-1734. doi: 10.1016/j.jacc.2017.01.051

15. Guglin M, Khan H. 2010. Pulmonary hypertension in heart failure. J Card Fail 16:461-474. doi: 10.1016/j.jacc.2017.-01.051 
16. Häggström J, Höglund K, Borgarelli M. 2009. An update on treatment and prognostic indicators in canine myxomatous mitral valve disease. J Small Anim Pract 50(Suppl 1): 25-33. doi: 10.1111/j.1748-5827.2009.00800.x

17. Häggström J, Kvart C, Pedersen HD. 2005. Acquired valvular heart disease. In: Ettinger S, Feldman E (eds). Textbook of veterinary internal medicine. $6^{\text {th }}$ ed. St. Louis, USA: Elsevier Saunders. p 1022-1039.

18. Hansson K, Häggström J, Kvart C, et al. 2002. Left atrial to aortic root indices using two-dimensional and M-mode echocardiography in Cavalier King Charles Spaniels with and without left atrial enlargement. Vet Radiol Ultrasoun 43: 568-575. doi: $10.1111 /$ j.1740-8261.2002.tb01051.x

19. Johnson L, Boon J, Orton EC. 1999. Clinical characteristics of 53 dogs with doppler-derived evidence of pulmonary hypertension: 1992-1996. J Vet Intern Med 13: 440-447. doi: 10.1111/j.19391676.1999.tb01461.x

20. Kellum HB, Stepien RL. 2007. Sildenafil citrate therapy in 22 dogs with pulmonary hypertension. J Vet Intern Med 21: 1258-1264. doi: 10.1111/j.19391676.2007.-tb01947.x

21. Lang RM, Badano LP, Mor-Avi V, Afilalo J, Armstrong A, Ernande L, Flachskampf $F$, et al. 2015. Recommendations for cardiac chamber quantification by echocardiography in adults: an update from the American Society of Echocardiography and the European Association of Cardiovascular Imaging. J Am Soc Echocardiog 28: 139. doi: 10.1016/j.echo.2014.10.003

22. Lankhaar JW, Westerhof N, Faes TJ, Gan CT, Marques KM, Boonstra A, van den Berg FG, et al. 2008. Pulmonary vascular resistance and compliance stay inversely related during treatment of pulmonary hypertension. Eur Heart J 29: 1688-1695. doi: 10.1093/ eurheartj/ehn103
23. MacDonald K, Johnson L. 2005. Pulmonary hypertension and pulmonary thromboembolism. In: Ettinger S, Feldman E (eds). Textbook of veterinary internal medicine. $6^{\text {th }}$ ed. St. Louis, USA: Elsevier Saunders. p 1284-1288.

24. Mikawa S, Miyagawa $Y$, Toda $N$, Tominaga Y, Takemura N. 2015. Predic-tive model for the detection of pulmonary hypertension in dogs with myxomatous mitral valve disease. J Vet Med Sci 77: 7-13. doi: 10.1292/jvms.140050

25. Olsen LH, Martinussen T, Pedersen HD. 2003. Early echocardiographic predictors of myxomatous mitral valve disease in Dachshunds. Vet Rec 152: 293-297. doi: 10.1136/vr.152.10.293

26. Pedersen HD, Lorentzen KA, Kristensen BO. 1999. Echocardiographic mitral valve prolapse in Cavalier King Charles spaniels: epidemiology and prognostic significance for regurgitation. Vet Rec 144: 315-320. doi: 10.1136/ vr.144.12.315

27. Ray L, Mathieu M, Jespers P, Hadad I, Mahmoudabady M, Pensis A, Motte $S$, et al. 2007. Early increase in pulmonary vascular reactivity with overexpression of endothelin-1 and vascular endothelial growth factor in canine experimental heart failure. Exp Physiol 93: 434.442. doi: 10.1113/ expphysiol.2007.040469

28. Rich S. 2001. Pulmonary hypertension. In: Braunwald E, Zipes DP, Libby P (eds). Heart disease: a textbook of cardiovascular medicine. Philadelphia, USA: WB Saunders. p 1908-1935.

29. Schober KE, Baade H. 2006. Doppler echocardiographic prediction of pulmonary hypertension in West Highland White Terriers with chronic pulmonary disease. J Vet Intern Med 20: 912-920. doi: 10.1111/j.1939-1676.2006.tb01805.x

30. Serres F, Chetboul V, Gouni V, Tissier $R$, Sampedrano CC, Pouchelon JL. 2007. Diagnostic value of echo-doppler and tissue doppler imaging in dogs with 
pulmonary arterial hypertension. J Vet Intern Med 21: 1280-1289. doi: 10.1111/ j.1939-1676.2007.tb01950.x

31. Serres FJ, Chetboul V, Tissier R, Sampedrano C, Gouni V, Nicolle AP, Pouchelon JL. 2006. Doppler echocardiography-derived evidence of pulmonary arterial hypertension in dogs with degenerative mitral valve disease: 86 cases (2001-2005). J Am Vet Med Assoc 229: 1772-1778. doi: 10.2460/ javma.229.11.1772

32. Stepien RL. 2009. Pulmonary arterial hypertension secondary to chronic leftsided cardiac dysfunction in dogs. J Small Anim Pract 50: 34-43. doi: 10.1111/ j.1748-5827.2009.00802.x

33. Talavera J, Fernández del Palacio J. 2007. Hipertensión pulmonar en perros y gatos. Clín Vet Peq Anim 27: 37-48.

34. Tidholm A, Höglund K, J. Häggström, Ljungvall I. 2015. Diagnostic value of selected echocardiographic variables to identify pulmonary hypertension in dogs with myxomatous mitral valve disease.
J Vet Intern Med 29: 1510-1517. doi: 10.1111/jvim. 13609

35. Vezzosi T, Mannucci T, Pistoresi A, Toma F, Tognetti R, Zini E, Domenech O, et al. 2017. Assessment of lung ultrasound B-lines in dogs with different stages of chronic valvular heart disease. J Vet Intern Med 31: 700-704. doi: 10.1111/jvim. 14692

36. Visser LC, Im MK, Johnson LR, Stern JA. 2016. Diagnostic value of right pulmonary artery distensibility index in dogs with pulmonary hypertension: comparison with Doppler echocardiographic estimates of pulmonary arterial pressure. J Vet Intern Med 30: 543-552. doi: 10.1111/jvim.13911

37. Ware W. 2007. Acquired valve diseases. In: Ware W. Cardiovascular disease in small animal medicine. London: Manson. p 263-279.

38. Webb JA, Armstrong J. 2002. Chronic idiopathic pulmonary fibrosis in a West Highland white terrier. Can Vet J 43: 703-705. 